\title{
Wo steht die kritische Stadtgeographie?
}

Rezension zu Bernd Belina / Matthias Naumann / Anke Strüver (Hg.) (2018):

Handbuch Kritische Stadtgeographie. Münster: Westfälisches Dampfboot.

Hanna Hilbrandt

Abb. 1 Titel des Buches (Quelle: Westfälisches Dampfboot)

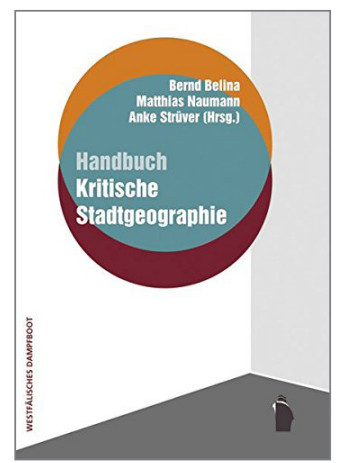

Das Handbuch Kritische Stadtgeographie ist Leser*innen dieser Zeitschrift schon aus einer 2015 erschienen Rezension der Erstauflage dieser Veröffentlichung bekannt (Beurskens 2015). Es spricht für die wachsende Bedeutung der kritischen Stadtgeographie, dass auch die 2016 erschienene zweite Auflage des Buches bald vergriffen war, und 2018 eine dritte, korrigierte und stark erweiterte Auflage neu herausgekommen ist. Mit dem Buch reagieren die Herausgeber*innen Bernd Belina, Matthias Naumann und Anke Strüver auf eine Leerstelle in der Geographielehre im Bereich dieses Themenfeldes. Ihre Antwort, eine „überblicksartige Darstellung der kritischen Stadtgeographie, ihrer zentralen Konzepte und empirischen Anwendungen in einer deutschsprachigen Einführung " (Belina/Naumann/ Strüver 2018: 16), richtet sich an Geographie-Studierende in den ersten Semestern. Zweifellos hat das Buch auch in vielen Bibliotheken benachbarter Disziplinen seinen festen Platz gefunden und wird auch höheren Semestern als Einführung dienen.

Was ist neu an der dritten Auflage? Das aktualisierte Handbuch wurde um 23 Kapitel auf nun 66 erweitert und deckt damit ein breiteres Themenspektrum ab. Einzelne Beiträge wurden überarbeitet. Ein zweiseitiges Vorwort der Herausgeber*innen skizziert die vorgenommenen thematischen Ergänzungen und begründet diese mit der Weiterentwicklung der Disziplin (2018: 11). Eine wünschenswerte Veröffentlichung als E-Book steht auch in der Neuauflage weiterhin aus.

Entsprechend des Schemas der bestehenden Beiträge zeichnen sich die ca. 5-seitigen neuen Kapitel durch anschauliche Fallbeispiele, eine zugängliche Sprache und brauchbare Leseempfehlungen aus. Meine Erfahrungen in der eigenen Unterrichtspraxis mit diesem Handbuch belegen bereits, dass diese Konzeption Studierenden einen leichten Einstieg in geographische Fragestellungen ermöglicht. Auch in der Neuerscheinung gliedert sich das Buch in fünf Teile, die Theorien, Methoden, Begriffe und Themen kritischer Stadtgeographie sowie ,Kämpfe in der Stadt' abbilden. Diese klare Strukturierung schafft Übersicht, ist aber nur hinsichtlich der ersten zwei Teile - Theorien und Methoden - schlüssig. Demgegenüber ist die Gliederung in 
Begriffe, Themen und Kämpfe der Stadt(-geographie) nicht nur, wie die Herausgeber*innen schreiben, „nicht immer trennscharf“ (Belina/Naumann/ Strüver 2018: 17), sondern in Ansätzen etwas willkürlich. Dennoch nimmt diese Strukturierung Setzungen vor, die jedoch unbegründet bleiben. Dass etwa Informalität hier ,nur' als ,Begriff' und nicht etwa als ,Kampf gewertet wird, wohl aber Urban Gardening in der letztgenannten Kategorie erscheint, deutet auf ein spezifisches Verständnis von Veränderungspotentialen (in) der Stadt, über das ich als Leserin gerne mehr erfahren hätte.

Zu den einzelnen Teilen: Dem Vorwort der neuen Auflage folgt eine kurze Einleitung in den thematischen Dreiklang Stadt, Kritik und Geographie sowie eine Erläuterung der Konzeption und des Aufbaus des Buches. Hier erfahren Leser*innen Einführendes über die Bedeutung des Städtischen, den Begriff der Kritik und die Einordnung des Buches innerhalb des Faches Geographie. Eine neue Generation von Stadtforscher*innen wird mit Interesse lesen, was ihre Forschung kritisch macht, nämlich nicht die Themensetzung sondern - so die Herausgeber*innen - eine Perspektive, die „Stadt und städtische Prozesse [...] als historisch gewordene und politisch veränderbare begreift und die in Stadt und städtische Entwicklungen mit emanzipatorischer Absicht eingreifen will“ (Belina/Naumann/Strüver 2018: 16). Insgesamt überzeugt die Einleitung eher durch Konzentration und Dichte als durch Ausführung und Detailliertheit. Sie verzichtet auf vertiefende Erläuterungen gegenwärtiger Strömungen, wichtiger Herausforderungen und zentraler Fragen kritischer Stadtgeographie. Damit entspricht das Einleitungskapitel den Anforderungen einer lexikalischen Nutzung des Buches. Um neuen Stadtgeograph*innen den Einstieg in das Feld zu erleichtern, wäre es darüber hinaus sicher sinnvoll, einzelne Kapitel zueinander in Beziehung zu setzen und aktuelle Debatten historisch zu kontextualisieren.

Teil I stellt kritische Theorien der Stadtforschung vor. Den ,Klassikern“ (unter anderem Marx und Engels, Lefebvre, Foucault, Harvey und Massey) und neueren Ansätzen (unter anderem zu Kritischem Weißsein und der postpolitischen Stadt), die bereits in der ersten Ausgabe reflektiert wurden, haben die Herausgeber*innen nun Kapitel über Bourdieu, Anarchismus sowie Affekte und Emotionen hinzugefügt. Dieser bereits in der letzten Auflage zurecht gelobte Teil (Adelhof 2015: 211) bietet in der Überarbeitung eine hervorragende Hilfe zum Einstieg in ein nun breiteres Spektrum städtischräumlicher Theorien.

Es lässt sich vermuten, dass die Themenauswahl dieses und anderer Buchteile allein durch die Herausgeber*innen getroffen wurde, deren Kompetenz für diese Aufgabe zweifellos überzeugt. Angesichts der politischen Brisanz jeder Form von Kanonisierung würde man sich jedoch wünschen, in der Einleitung oder in denkbaren thematischen Einführungen in die Kapitelblöcke eine transparente Begründung der Entscheidungskriterien dieser Zusammenstellung zu lesen. Warum finden in diesem who's who der Stadtforschung beispielsweise Walter Benjamin, Manuel Castells, Iris Marion Young, Akteur-Netzwerk-Theorie, Pragmatismus und postkoloniale Theorien keinen Platz? Dass Doreen Massey hier immer noch alleine den Platz weiblicher Theoretikerinnen verteidigen muss, ist leicht durch deren Marginalisierung in der Disziplin begründet. Jedoch verpasst das Kapitel die Chance kritische raum- oder materialitäts-interessierte Stadtforscherinnen 
in den Kanon der Ausgewählten aufzunehmen. Meine Vorschlagsliste Dolores Hayden, Saskia Sassen, Jennifer Robinson, Ananya Roy, Donna Haraway, Nancy Fraser oder Iris Marion Young - können Andere sicher sinnvoll erweitern.

Teil II widmet sich Methoden der Stadtforschung. Der Kritik der „Einseitigkeit“" (Beurskens 2015: 175) dieses Buchteils und dem Wunsch nach einer „breitere(n) Palette gängiger sowie seltenerer/neuerer Methoden“ (ebd.) begegnet die Neuauflage sinnvoll mit einer Erweiterung des dargestellten Spektrums um ethnographische, visuelle, rekonstruktive und geodatengestützte Methoden. Zusammen mit den bestehenden Kapiteln zu Datenerhebungs- und Analyseverfahren (unter anderem „Kritische Kartographien der Stadt“, „Die Stadt im Interview“ und „Aktivistische Stadtforschung"), lässt sich dieser Buchteil nun fast als ein dünnes Methodenhandbuch nutzen. Auch um Platz für weitere theoretische oder thematische Kapitel zu schaffen, würde es sich anbieten, diesen Teil in der nächsten Auflage erweitert als Handbuch Methoden Kritischer Stadtgeographie auszukoppeln.

Es ist der Vielfalt kritischer Methoden geschuldet, dass sich hier dennoch weiterbestehende Lücken aufzeigen lassen. Aber auch in diesem Buchteil hätte eine Begründung der Grenzen der getroffenen Auswahl offenlegen können, warum etwa Archivdaten, Fragebögen oder Fokusgruppen in diesem kritischen Methodenkanon keinen Platz finden. Unbeantwortet bleibt auch die Frage, ,inwiefern eine kritische Stadtgeographie sich methodisch von jeglicher guter Wissenschaft unterscheidet" (Basten 2015: 120). Die Herausgeber*innen formulieren in der Einleitung, es gälte „scheinbar rein technische[...] und regelgeleitete[...] methodische[...] Aspekte der Stadtgeographie zu hinterfragen und sie in Hinblick auf (selbst-)kritische Anwendung zu reformulieren" (Belina/Naumann/Strüver 2018: 17). Statt es den Autor*innen zu überlassen, diese Aussage mit Inhalten zu füllen, ließen sich zum Beispiel in einer Einleitung in diesen Buchteil die Auswahl der besprochenen Methoden, wesentliche Aspekte einer kritischen Methodennutzung sowie forschungsethische Fragen einer engagierten Stadtforschung erläutern.

Die Teile III, IV und V erläutern Begriffe, Themen und Kämpfe der Stadt(-geographie) durch eine erweiterte Auswahl aktueller stadträumlicher Fragestellungen. Darunter fallen Themen der Sozial- und Kulturgeographie, etwa in den Kapiteln „Körper“, „Bildung“ oder „Queer“, polit-ökonomische Einführungen in Immobilienmärkte, Gentrification oder die unternehmerische Stadt sowie Kapitel zu Themenstellungen feministischer Debatten. Die Neuauflage erweitert dieses Spektrum um Ansätze, die seit der Erstauflage vermehrt im Zentrum kritischer Forschung und Praxis stehen. Der Einfluss des material turns zeigt sich hier wirkmächtig in Kapiteln zu Ernährung, Gesundheit, Architektur, oder der Smart City; deutlicher sogar als die wachsende Bedeutung von Rechtsruck und Migration, die sich in Kapiteln zu Urban Citizenship und Racial Profiling niederschlägt. Aufregend ist die Vielzahl neuer Kapitel zu Kapitalismusalternativen, etwa zu Munizipalismen, den Commons, Alternativem Wirtschaften, hier auch: Urban Citizenship. Zudem ist die Erweiterung um polit-ökonomische Themen, unter anderem unter den Titeln „Tourismus“, „kommunale Austerität“, „Gewerkschaften“ und „Kreative Stadt“ nennenswert. Diese Kapitel ergänzen sich sinnvoll 
und sind durch Quervereise hilfreich verknüpft. Einzig ein Glossar könnte die hervorragende Zugänglichkeit des Stoffes weiter verbessern. Insgesamt liegt der große Wert dieser Buchteile in der Leichtigkeit, mit der sie ihren Leser*innen neue Themenbereiche erschließen und sie zur weiterführenden Lektüre inspirieren.

Abschließend die Gretchenfrage: Stehen aktuelle Debatten der kritischen Stadtgeographie dort, wo sie die Macher*innen dieses Handbuchs sehen? Ja und nein. In der neuen Auflage deckt das Handbuch ein weites Spektrum zentraler Themen und Diskussionen ab. Wie die Herausgeber*innen aber selber schreiben (2018: 12), bleibt ,auch diese Auflage des Handbuchs unvollständig und bedarf einer steten Aktualisierung um weitere Einträge“. So erfahren wir, wie Städte vermarktet, genutzt und kontrolliert werden, nicht aber wie sie produziert, also physisch gebaut werden. Es fehlen dem Handbuch Begriffe der Finanzialisierung, sowie legale und temporale Zugänge. Leerstellen in den Themenbereichen Eliten, Wahlen, Religionen und politische Parteien legen nah, dass vor allem für eine Geographie der neuen Rechten in der nächsten Ausgabe Platz geschaffen werden muss.

Meine Befürchtung, dass die Selbsternennung zur kritischen Stadtforschung nur das Angebot einer relativ geschlossenen Community reproduziert, die hier mit- und füreinander schreibt, löst sich bei genauerer Durchsicht der Themenvielfalt des Buches und der Vielstimmigkeit der Autor*innen schnell auf. Es bleibt jedoch der Eindruck, dass die Vorstellung von emanzipatorischen Veränderungen, die vor allem den letzten Buchteil trägt, sich auf ein paar wenige, kostbare und vornehmlich aktivistische Handlungen und deren favorisierte Orte fokussiert und damit beispielsweise stille Unterwanderungspraxen, routinierte Aushandlungen und Alltäglichkeit als Schauplatz progressiver Kritik außer Acht lässt. Dazu trägt bei, dass die Kapitel sich vornehmlich auf empirische Fallbeispiele aus Deutschland beschränken (Belina/Naumann/ Strüver 2018: 18) - eine disputable Entscheidung, die die Herausgeber*innen mit deren besserer Zugänglichkeit begründen, die aber in der Konsequenz eine Reihe empirischer Forschungsgegenstände und in der Folge auch bestimmte Fragstellungen ins Abseits schiebt. Glücklicherweise gibt es einige Kapitel, die dieser Setzung nicht nachkommen, so dass das Buch insgesamt eine interdisziplinäre und breite Themenpalette vorstellt, die über das begrüBenswerte Ziel, der kritischen Stadtforschung in der Geographielehre auf den Sprung zu helfen, weit hinausgeht. Indem dieses eindrucksvolle Handbuch die wachsende Bedeutung der kritischen Stadtforschung in der deutschsprachigen Stadtforschung dokumentiert, zeugt es bereits jetzt von deren Vielseitigkeit, Institutionalisierung und Professionalisierung über disziplinäre Grenzen hinweg.

\section{Autor_innen}

Hanna Hilbrandt ist Stadtgeographin und Architektin. Sie arbeitet zu Informalität, Wohnungspolitik, städtischer Governance, Finanzialisierung und Migration.

hanna.hilbrandt@complicity.de 


\section{Literatur}

Adelhof, Katja (2015): Rezension: Bernd Belina, Matthias Naumann, Anke Strüver (Hrsg.): Handbuch Kritische Stadtgeographie. In: die Erde 146/2-3: 211-212.

Basten, Ludger (2015): Rezension Handbuch Kritische Stadtgeographie. In: Geographische Zeitschrift 103/2: 119 -122.

Belina, Bernd / Naumann, Matthias / Strüver, Anke (Hg.) (2018): Handbuch Kritische Stadtgeographie. Münster: Westfälisches Dampfboot.

Belina, Bernd / Naumann, Matthias / Strüver, Anke (2018): Stadt, Kritik und Geographie. Einleitung zum Handbuch Kritische Stadtgeographie. In: Bernd Belina / Matthias Naumann / Anke Strüver (Hg.): Handbuch Kritische Stadtgeographie. Münster: Westfälisches Dampfboot, 14-19.

Beurskens, Kristine (2015): Kritisches Denken aufbereitet. Rezension zu Bernd Belina, Matthias Naumann, Anke Strüver (Hg.) (2014): Handbuch Kritische Stadtgeographie. In: $\mathrm{s}$ u b $\backslash \mathrm{u}$ r b a n. zeitschrift für kritische stadtforschung 3/2: 167-170. 